Bruno Girotto and Silvano Holzer, Dipartimento di Matematica Applicata "B. de Finetti", University of Trieste, Piazzale Europa 1, I-34127 Trieste, Italy. e-mail: brunog@econ.univ.trieste.it, holzer@univ.trieste.it

\title{
WEAK CONVERGENCE OF BOUNDED, MONOTONE SET FUNCTIONS IN AN ABSTRACT SETTING
}

\begin{abstract}
We introduce an abstract treatment of the weak convergence for bounded monotone set functions which allows us to obtain some basic results generalizing well known theorems regarding classical weak and vague convergence and weak convergence of masses on normal topological spaces (e.g. Portmanteau type theorems, Direct and Converse Prokhorov type theorems). Moreover, we introduce a suitable topology (called the Lévy-topology) in order to study the properties of this abstract convergence from a topological point of view.
\end{abstract}

Dedication This article is dedicated, with a deep sense of loss and wonder, to the memory of our teacher, our friend, our colleague, Luciano Daboni.

\section{Introduction}

It is well known that the notion of weak convergence of measures plays a key role in the classical $\sigma$-additive treatment of probability and stochastic processes; see, for instance, Billingsley [3]. In this framework, Varadarajan [10] supplied the basic properties of the weak convergence, especially from a topological point of view. In a finitely additive setting, after the pioneering work of Alexandroff [1], Masani [9] first extended the direct Prokhorov theorem to the space of outer regular masses on normal Hausdorff topological spaces and

\footnotetext{
Key Words: Monotone set function, Choquet integral, weak convergence, Lévy-topology, tightness.

Mathematical Reviews subject classification: Primary 28A12, 28A33; Secondary 60B10, 60B05

Received by the editors July 25, 1999

* Research supported by the National Group "Statistical Models: Probabilistic Foundations and Procedures for Inference and Decisions".
} 
Girotto and Holzer $[6,7,8]$ extended the Portmanteau theorem and Masani results to the space of masses (outer regular or not) on normal topological spaces.

On the other hand, over the last four decades, there has been a sustained growth of interest in monotone set functions (non necessarily additive) for the treatment of uncertainty. The mathematical theory of monotone set functions got its first important contribution with Gustave Choquet (1953), who was concerned in statistical mechanics and potential theory. Monotone set functions were also considered in the seminal contribution of Lloyd Shapley (1953) in the study of cooperative games. Some years later, the statistician Arthur Dempster (1966) first presented an application of monotone set functions in the field of Bayesian inference. Decision theorists rediscovered non-additivity when David Schmeidler (1989) first introduced an axiomatic model of choice with non-additive beliefs. Recently, other fields of applications of non-additive set functions are, for instance, artificial intelligence, finance and asset pricing theory.

Motivated by these reasons and by its interest from a mathematical point of view, in this paper we introduce the notion of weak convergence of bounded monotone set functions (additive or not) and extend the Portmanteau theorem and Prokhorov theorem in this general framework.

Now, we briefly describe the contents of the following sections. In Section two, we introduce the notion of regular and strongly regular sets, which are the main tool for studying the weak convergence of bounded monotone set functions. In Section three, we introduce this convergence and prove a Portmanteau type theorem, which characterizes it. In Section four we introduce the Lévy-topology and supply a Prokhorov type theorem, both in direct form and converse one. An appendix concludes the paper by recalling some properties of the Choquet integral which are instrumental in getting some of our results.

\section{The Regularity Systems}

Throughout this paper we adopt usual set theoretic and topological notation. Given a non-empty set $\Omega$, the letter $\mathcal{F}$ always denotes a family of subsets of $\Omega$ including $\emptyset$ and closed under complementation; sets from $\mathcal{F}$ are denoted by $F$, with or without indices.

The letters $\mathcal{C}, \mathcal{U}$ always denote two subsets of $\mathcal{F}$ such that $\emptyset \in \mathcal{C}$ and $\Omega \in \mathcal{U}$ (i.e. $\mathcal{C}, \mathcal{U}$ are a $(\emptyset)$-paving and a $(\Omega)$-paving, respectively); moreover, $C$ and $U$, with or without indices, are elements of $\mathcal{C}$ and $\mathcal{U}$, respectively.

We denote by $\mu$, with or without indices, any bounded (i.e. finite) mono- 
tone set function on $\mathcal{F}$ with zero value at the empty set; moreover, $\|\mu\|$ is the norm of $\mu$, i.e. $\|\mu\|=\mu(\Omega)$. As usual, for any $\mu$, the set function $\bar{\mu}$ on $\mathcal{F}$ is the conjugate set function of $\mu$, i.e. $\bar{\mu}(F)=\|\mu\|-\mu\left(F^{c}\right)$ for all $F$.

Now, we introduce two basic notions of regularity for a bounded monotone set function on $\mathcal{F}$.

Definition 2.1. We say that $F$ is a:

- $\mu$-regular set (w.r.t. $(\mathcal{C}, \mathcal{U}))$ iff it satisfies the "approximation property":

$$
\sup \{\mu(C): C \subset F\}=\mu(F)=\inf \{\mu(U): U \supset F\},
$$

i.e. for all $\epsilon>0$ there are $C \subset F$ and $U \supset F$ such that $\mu(U)-\mu(C)<\epsilon$. We denote by $\mathcal{R}_{\mu}$ the set of $\mu$-regular sets and we call it the $\mu$-regularity system;

- $\mu$-strongly regular set (w.r.t. $(\mathcal{C}, \mathcal{U}))$ iff for all $\epsilon>0$ there are $C, U \in \mathcal{R}_{\mu}$ such that $U \subset F \subset C$ and $\mu(C)-\mu(U)<\epsilon$. We denote by $\mathcal{R}_{\mu}^{0}$ the set of $\mu$-strongly regular sets and we call it the $\mu$-strong regularity system.

Remark 2.2. (i) Let $\mathcal{C}=\mathcal{U}^{c}=\left\{U^{c}: U \in \mathcal{U}\right\}$. Then, one can easily prove that $F \in \mathcal{R}_{\mu} \Longleftrightarrow F^{c} \in \mathcal{R}_{\bar{\mu}}$ and $F \in \mathcal{R}_{\mu}^{0} \Longleftrightarrow F^{c} \in \mathcal{R}_{\bar{\mu}}^{0}$. Consequently, if $\mu$ is additive, $\mathcal{R}_{\mu}$ and $\mathcal{R}_{\mu}^{0}$ are closed under complements, on noting that $\mu=\bar{\mu}$.

(ii) Let $\Omega$ be a topological space, $\mathcal{F}$ a field, $\mathcal{C}$ the family of closed sets, $\mathcal{U}$ the family of open sets and $\mu$ be additive. Then the $\mu$-strong regularity system is the family of sets with $\mu$-null and $\mu$-regular boundary. Indeed, given $F \in \mathcal{R}_{\mu}^{0}$, let $\epsilon>0$. Then, there are $C, U \in \mathcal{R}_{\mu}$ such that $U \subset F \subset C$ and $\mu(C)-\mu(U)<\epsilon$. Moreover, there are $C^{\prime} \subset U$ and $U^{\prime} \supset C$ such that $\mu(U)-\mu\left(C^{\prime}\right)<\epsilon$ and $\mu\left(U^{\prime}\right)-\mu(C)<\epsilon$. By the additivity of $\mu$, we have $\mu\left(U^{\prime}-C^{\prime}\right)=\mu\left(U^{\prime}\right)-\mu\left(C^{\prime}\right)$ and hence $\mu\left(U^{\prime}-C^{\prime}\right)<3 \epsilon$. On noting that the boundary $\partial F$ of $F$ is a subset of the closed set $C-U \subset U^{\prime}-C^{\prime} \in \mathcal{U}$, it follows that $\mu(\partial F) \leq \mu\left(U^{\prime}-C^{\prime}\right)<3 \epsilon$. Consequently, since $\epsilon$ is arbitrarily chosen, $\partial F$ is $\mu$-null and $\mu$-regular. Conversely, by Theorem 2.5(ii) in Girotto and Holzer [7], one can easily prove that any set with $\mu$-null and $\mu$-regular boundary is an element of $\mathcal{R}_{\mu}^{0}$. Therefore, the notion of $\mu$-strong regularity here considered can be seen as a generalization of the one given in Girotto and Holzer [7].

The following theorem assures that the latter notion of regularity in Definition 2.1 is a strengthening of the former.

Theorem 2.3. We have: $\mathcal{R}_{\mu}^{0} \subset \mathcal{R}_{\mu}$.

Proof. Given a $\mu$-strongly regular set $F$, let $\epsilon>0$. Then there are $C, U \in \mathcal{R}_{\mu}$ such that $U \subset F \subset C$ and $\mu(C)-\mu(U)<\epsilon$. Moreover, there are $C^{\prime} \subset U$ and 
$U^{\prime} \supset C$ such that $\mu(U)-\mu\left(C^{\prime}\right)<\epsilon$ and $\mu\left(U^{\prime}\right)-\mu(C)<\epsilon$. Therefore, we have $C^{\prime} \subset F \subset U^{\prime}$ and $\mu\left(U^{\prime}\right)-\mu\left(C^{\prime}\right)<3 \epsilon$.

The following definition introduces the notion of measurable function w.r.t. the pair $(\mathcal{C}, \mathcal{U})$ and allows us to specify some interesting $\mu$-strongly regular sets. To this end, given a real map $f$ on $\Omega$, we put:

$$
\{f>t\}=\{\omega \in \Omega: f(\omega)>t\}, \quad\{f \geq t\}=\{\omega \in \Omega: f(\omega) \geq t\},
$$

for any real number $t$.

Definition 2.4. We say that a real map $f$ on $\Omega$ is $(\mathcal{C}, \mathcal{U})$-measurable iff:

- for any $t>0$ we have $\{f \geq t\} \in \mathcal{C}$ and $\{f>t\} \in \mathcal{U}$, if $f \geq 0$;

- $f^{+}$and $f^{-}$are $(\mathcal{C}, \mathcal{U})$-measurable, if $f$ is arbitrary.

We denote by $\mathbf{M}(\mathcal{C}, \mathcal{U})$ the set of $(\mathcal{C}, \mathcal{U})$-measurable maps.

Remark 2.5. Since we want to consider $(\mathcal{C}, \mathcal{U})$-measurable functions also when $\Omega \notin \mathcal{C}$ (see, for instance, Remark 3.2(ii)), we do not allow $t=0$ in the previous definition. In the contrary case, indeed, we should have $\Omega \in \mathcal{C}$ whenever $\mathbf{M}(\mathcal{C}, \mathcal{U}) \neq \emptyset$.

Theorem 2.6. Given $f \in \mathbf{M}(\mathcal{C}, \mathcal{U})$, let $G_{\mu}(t)=\mu(\{f>t\})$ and $H_{\mu}(t)=$ $\mu(\{f \geq t\})$ for any $t>0$. If $t$ is a continuity point of $G_{\mu}$, then $\{f \geq t\}$ and $\{f>t\}$ are $\mu$-strongly regular sets; moreover $G_{\mu}(t)=H_{\mu}(t)$ and $t$ is a continuity point of $H_{\mu}$, as well.

Proof. Let $t>0$ be a continuity point of $G_{\mu}$. First, we prove that $\{f \geq t\}$ and $\{f>t\}$ are $\mu$-regular sets. Let $\left(x_{n}\right)$ and $\left(y_{n}\right)$ be real strictly monotone sequences such that $x_{n} \uparrow t$ and $y_{n} \downarrow t$. Then, for any $n$, we have:

$$
\left\{f>y_{n}\right\} \subset\left\{f \geq y_{n}\right\} \subset\{f>t\} \subset\{f \geq t\} \subset\left\{f \geq x_{n+1}\right\} \subset\left\{f>x_{n}\right\} .
$$

On noting that the sets considered in the previous relation are elements of $\mathcal{F}$ and recalling that $t$ is a continuity point of $G_{\mu}$, we get $\mu\left(\left\{f>x_{n}\right\}\right)-\mu(\{f>$ $\left.\left.y_{n}\right\}\right)=G_{\mu}\left(x_{n}\right)-G_{\mu}\left(y_{n}\right) \rightarrow 0$ and hence $\{f>t\},\{f \geq t\} \in \mathcal{R}_{\mu}$ (note that $\left\{f \geq y_{n}\right\} \in \mathcal{C}$ and $\left\{f>x_{n}\right\} \in \mathcal{U}$ for all $\left.n\right)$. Moreover, $G_{\mu}(t)=H_{\mu}(t)$. Looking again at the above inclusions, it is easily seen that $t$ is a continuity point of $H_{\mu}$, as well.

Finally, to verify that $\{f>t\}$ and $\{f \geq t\}$ are $\mu$-strongly regular sets it is enough to observe that $\{f>t\} \subset\{f \geq t\},\{f \geq t\} \in \mathcal{C},\{f>t\} \in \mathcal{U}$ and $\mu(\{f>t\})=\mu(\{f \geq t\})$. 
Corollary 2.7. Let $f \in \mathbf{M}(\mathcal{C}, \mathcal{U})$. Then there are at most a countable set of positive real numbers $t>0$ such that $\{f \geq t\}$ and $\{f>t\}$ are not $\mu$-strongly regular sets.

\section{Weak Convergence}

Given the set

$$
\mathbf{M}_{0, b}(\mathcal{C}, \mathcal{U})=\{f \in \mathbf{M}(\mathcal{C}, \mathcal{U}): \exists k(\sup |f| \leq k) \wedge \exists C \forall \omega(\omega \notin C \Rightarrow f(\omega)=0)\},
$$

collecting bounded $(\mathcal{C}, \mathcal{U})$-measurable maps which vanish outside elements of $\mathcal{C}$ (i.e. with support in $\mathcal{C}$ ), in what follows we assume that the pair $(\mathcal{C}, \mathcal{U})$ satisfies the following:

Separation Property: for any $C$ and $U$ such that $C \subset U$ there is $f \in$ $\mathbf{M}_{0, b}(\mathcal{C}, \mathcal{U})$ taking on values in $[0,1]$ such that $f(\omega)=1$, if $\omega \in C$, and $f(\omega)=0$, if $\omega \notin U$.

Since the separation property assures the set $\mathbf{M}_{0, b}(\mathcal{C}, \mathcal{U})$ is never empty (take $C=\emptyset$ and $U=\Omega$ ), we have $\emptyset \in \mathcal{U}$ and hence the empty set is $\mu$-strongly regular. Moreover, if $\mathcal{C}=\mathcal{U}^{c}$, we have $\Omega \in \mathcal{C}$ so that the set $\Omega$ is $\mu$-strongly regular, as well.

By the following definition we introduce the notion of weak convergence w.r.t. the pair $(\mathcal{C}, \mathcal{U})$ for bounded monotone set functions. In the sequel, for any $\mu$ and $f$, the symbol $\int f d \mu$ always denotes the Choquet integral (note that any $f \in \mathbf{M}_{0, b}(\mathcal{C}, \mathcal{U})$ is $\mu$-integrable for all $\mu$ ). Moreover, the convergence of a real net $\left\{r_{d} ; d \in D\right\}$ to the real number $r$ is denoted, henceforth, by $r_{d} \rightarrow r$.

Definition 3.1. We say that the net $\left\{\mu_{d} ; d \in D\right\}$ is weakly convergent to $\mu$ (w.r.t. $(\mathcal{C}, \mathcal{U})$ ), and write $\mu_{d} \stackrel{w}{\rightarrow} \mu$, iff $\int f d \mu_{d} \rightarrow \int f d \mu$ for any $f \in \mathbf{M}_{0, b}(\mathcal{C}, \mathcal{U})$.

Remark 3.2. (i) Let $\Omega$ be a normal space and $\mathcal{C}, \mathcal{U}$ the families of closed and open sets, respectively. Then, $\mathbf{M}_{0, b}(\mathcal{C}, \mathcal{U})$ is the set of bounded continuous real functions so that, by Urysohn Lemma, the pair $(\mathcal{C}, \mathcal{U})$ satisfies the separation property. Therefore, in the setting of bounded additive set functions $\mu$ on a field, the weak convergence w.r.t. $(\mathcal{C}, \mathcal{U})$ is the usual one, since the Choquet integral and the S-integral of a bounded continuous real function coincide (see Theorem A.11 in Appendix).

(ii) Let $\Omega$ be a locally compact Hausdorff space and $\mathcal{C}, \mathcal{U}$ the families of compact and open sets, respectively. Then, $\mathbf{M}_{0, b}(\mathcal{C}, \mathcal{U})$ is the set of continuous 
real functions with compact support so that, by Theorem 3.3.2 and Theorem 3.3.3 in Engelking [5], the pair $(\mathcal{C}, \mathcal{U})$ satisfies the separation property. Therefore, in the setting of bounded additive set functions $\mu$ on a field, the weak convergence w.r.t. $(\mathcal{C}, \mathcal{U})$ is the vague convergence.

Now, we are proving a Portmanteau type theorem, which gives a comprehensive list of conditions equivalent to the weak convergence; thus, we link weak convergence and pointwise convergence. To this end, first we supply the following result.

Lemma 3.3. Let $C \subset U$. Then there are $C^{\prime}, U^{\prime} \in \mathcal{R}_{\mu}^{0} \cap \mathcal{R}_{\mu}^{0}$ such that $C \subset$ $U^{\prime} \subset C^{\prime} \subset U$.

Proof. By the separation property, there is $f \in \mathbf{M}_{0, b}(\mathcal{C}, \mathcal{U})$ such that $f$ : $\Omega \mapsto[0,1]$ and $f(\omega)=1$, if $\omega \in C$, and $f(\omega)=0$, if $\omega \notin U$. Now, by Corollary 2.7, there is $t \in] 0,1\left[\right.$ such that $C^{\prime}=\{f \geq t\}$ and $U^{\prime}=\{f>t\}$ are elements of $\mathcal{R}_{\mu}^{0} \cap \mathcal{R}_{\bar{\mu}}^{0}$. Since $C \subset U^{\prime} \subset C^{\prime} \subset U$ we get the thesis.

Theorem 3.4. (Characterization Theorem) Given $\mu$ and a net $\left\{\mu_{d} ; d \in D\right\}$, the following statements are equivalent:

(i) $\mu_{d} \stackrel{w}{\rightarrow} \mu$;

(ii) $\bar{\mu}_{d} \stackrel{w}{\rightarrow} \bar{\mu}$;

(iii) $\limsup \sup _{d \in D} \mu_{d}(C) \leq \mu(C), \liminf _{d \in D} \mu_{d}(U) \geq \mu(U)$ for all $C, U \in \mathcal{R}_{\mu}$ and

$\lim \sup _{d \in D} \bar{\mu}_{d}(C) \leq \bar{\mu}(C), \liminf _{d \in D} \bar{\mu}_{d}(U) \geq \bar{\mu}(U)$ for all $C, U \in \mathcal{R}_{\bar{\mu}} ;$

(iv) $\mu_{d}(F) \rightarrow \mu(F)$ for all $F \in \mathcal{R}_{\mu}^{0}$ and $\bar{\mu}_{d}(F) \rightarrow \bar{\mu}(F)$ for all $F \in \mathcal{R}_{\bar{\mu}}^{0}$;

(v) $\left(\mu_{d}(U), \bar{\mu}_{d}(U)\right) \rightarrow(\mu(U), \bar{\mu}(U))$ for all $U \in \mathcal{R}_{\mu}^{0} \cap \mathcal{R}_{\bar{\mu}}^{0}$;

(vi) $\left(\mu_{d}(C), \bar{\mu}_{d}(C)\right) \rightarrow(\mu(C), \bar{\mu}(C))$ for all $C \in \mathcal{R}_{\mu}^{0} \cap \mathcal{R}_{\bar{\mu}}^{0}$.

Proof. (i) $\Longleftrightarrow$ (ii). It is enough to observe that, for any $f \in \mathbf{M}_{0, b}(\mathcal{C}, \mathcal{U})$, we have $-f \in \mathbf{M}_{0, b}(\mathcal{C}, \mathcal{U})$ and, by Theorem A.4(iii), $\int f d \bar{\mu}=-\int(-f) d \mu$.

(i) $\Rightarrow$ (iii). First, assume $C \in \mathcal{R}_{\mu}$. Let $\epsilon>0$. Then there is $U \supset C$ such that $\mu(U)-\mu(C)<\epsilon$. By the separation property we can consider $f \in \mathbf{M}_{0, b}(\mathcal{C}, \mathcal{U})$ such that $f: \Omega \mapsto[0,1]$ and $f(\omega)=1$, if $\omega \in C$, and $f(\omega)=0$, if $\omega \notin U$. Therefore, by Theorem A.4(i),(v), we have $\int f d \mu \leq \int 1_{U} d \mu=\mu(U)$ and $\mu_{d}(C)=\int 1_{C} d \mu_{d} \leq \int f d \mu_{d}$, for any $d \in D$. Hence we get

$$
\limsup _{d \in D} \mu_{d}(C) \leq \limsup _{d \in D} \int f d \mu_{d}=\int f d \mu \leq \mu(U)<\mu(C)+\epsilon,
$$


where the equality holds by (i). Since $\epsilon$ is arbitrary, we get $\lim \sup _{d \in D} \mu_{d}(C) \leq$ $\mu(C)$, as desired.

Now, assume $U \in \mathcal{R}_{\mu}$. Let $\epsilon>0$. Then there is $C \subset U$ such that $\mu(U)-\mu(C)<\epsilon$. By the separation property we can consider $f \in \mathbf{M}_{0, b}(\mathcal{C}, \mathcal{U})$ such that $f: \Omega \mapsto[0,1]$ and $f(\omega)=1$, if $\omega \in C$, and $f(\omega)=0$, if $\omega \notin U$. Therefore, we have $\mu(C) \leq \int f d \mu$ and $\int f d \mu_{d} \leq \mu_{d}(U)$, for any $d \in D$. Hence we get

$$
\liminf _{d \in D} \mu_{d}(U) \geq \liminf _{d \in D} \int f d \mu_{d}=\int f d \mu \geq \mu(C)>\mu(U)-\epsilon,
$$

where the equality holds by (i). Since $\epsilon$ is arbitrary, we get $\liminf _{d \in D} \mu_{d}(U) \geq$ $\mu(U)$, as desired.

Finally, keeping in mind the above proved equivalence (i) $\Longleftrightarrow$ (ii), one easily gets the remaining part of (iii). This proves the desired implication.

(iii) $\Rightarrow$ (iv). Let $F \in \mathcal{R}_{\mu}^{0}$. Given $\epsilon>0$, there are $C, U \in \mathcal{R}_{\mu}$ such that $U \subset F \subset C$ and $\mu(C)-\mu(U)<\epsilon$. Since $\mu(U) \leq \mu(F) \leq \mu(C)$ and $\mu_{d}(U) \leq \mu_{d}(F) \leq \mu_{d}(C)$ for any $d \in D$, we have

$$
\begin{aligned}
\mu(U) & \leq \liminf _{d \in D} \mu_{d}(U) \leq \liminf _{d \in D} \mu_{d}(F) \\
& \leq \limsup _{d \in D} \mu_{d}(F) \leq \limsup _{d \in D} \mu_{d}(C) \leq \mu(C)<\mu(U)+\epsilon
\end{aligned}
$$

and hence $\mu(F), \liminf \operatorname{in}_{d \in D} \mu_{d}(F), \lim \sup _{d \in D} \mu_{d}(F) \in[\mu(U), \mu(U)+\epsilon]$. Since $\epsilon$ is arbitrarily chosen, we get $\mu_{d}(F) \rightarrow \mu(F)$. This proves the former part of (iv). Similarly, one can prove the latter part of (iv).

(iv) $\Rightarrow(\mathrm{v})+(\mathrm{vi})$. This is obvious.

(v) $\Rightarrow$ (i). Let $f \in \mathbf{M}_{0, b}(\mathcal{C}, \mathcal{U})$. First, assume $0 \leq f \leq k$ and $C$ be such that $f(\omega)=0$ for any $\omega \notin C$. Since $\Omega \in \mathcal{U}$, by Lemma 3.3, there is $U \in \mathcal{R}_{\mu}^{0} \cap \mathcal{R}_{\mu}^{0}$ such that $C \subset U$. Hence, by $(\mathrm{v}), \mu_{d}(U) \rightarrow \mu(U)$ and $\bar{\mu}_{d}(U) \rightarrow \bar{\mu}(U)$. Let $\epsilon>0$. Then, by Corollary 2.7, there are real numbers $t_{0}, t_{1}, \ldots, t_{m}$ such that $t_{0}=0<t_{1}<\cdots<t_{m-1}<k \leq t_{m}$ and

$$
t_{i}-t_{i-1}<\epsilon, \quad U_{i}=\left\{f>t_{i}\right\} \in \mathcal{R}_{\mu}^{0} \cap \mathcal{R}_{\bar{\mu}}^{0} \quad(i=1, \ldots, m) .
$$

Then, by $(\mathrm{v}), \mu_{d}\left(U_{i}\right) \rightarrow \mu\left(U_{i}\right)$ and $\bar{\mu}_{d}\left(U_{i}\right) \rightarrow \bar{\mu}\left(U_{i}\right)$ for any $i=1, \ldots, m$.

Letting $g_{\epsilon}=\sum_{i=1}^{m}\left(t_{i}-t_{i-1}\right) 1_{U_{i}}$ and noting that $f(\omega)=0$ for any $\omega \notin U$, we have $g_{\epsilon} \leq f \leq g_{\epsilon}+\epsilon 1_{U}$. Moreover, by Remark A.2(i), the maps $g_{\epsilon}$ and $g_{\epsilon}+\epsilon 1_{U}$ are simple functions (note $U \supset U_{1} \supset \cdots \supset U_{m}$ ). Then, by Theorem 
A.4(v) and Remark A.9, we have

$$
\begin{aligned}
\int g_{\epsilon} d \mu_{d} & \leq \int f d \mu_{d} \leq \int\left(g_{\epsilon}+\epsilon 1_{U}\right) d \mu_{d}=\sum_{i=1}^{m}\left(t_{i}-t_{i-1}\right) \mu_{d}\left(U_{i}\right)+\epsilon \mu_{d}(U) \\
& =\int g_{\epsilon} d \mu_{d}+\epsilon \mu_{d}(U)
\end{aligned}
$$

for any $d \in D$, and

$$
\int g_{\epsilon} d \mu \leq \int f d \mu \leq \int g_{\epsilon} d \mu+\epsilon \mu(U)
$$

moreover, keeping in mind that $\mu_{d}\left(U_{i}\right) \rightarrow \mu\left(U_{i}\right)$ for any $i=1, \ldots, m$, we have

$$
\int g_{\epsilon} d \mu_{d}=\sum_{i=1}^{m}\left(t_{i}-t_{i-1}\right) \mu_{d}\left(U_{i}\right) \rightarrow \sum_{i=1}^{m}\left(t_{i}-t_{i-1}\right) \mu\left(U_{i}\right)=\int g_{\epsilon} d \mu .
$$

Consequently, recalling that $\mu_{d}(U) \rightarrow \mu(U)$, by carrying out the passage to the limit, we get

$$
\int g_{\epsilon} d \mu \leq \liminf _{d \in D} \int f d \mu_{d} \leq \limsup _{d \in D} \int f d \mu_{d} \leq \int g_{\epsilon} d \mu+\epsilon \mu(U)
$$

and hence, from (1), we have

$$
\left|\liminf _{d \in D} \int f d \mu_{d}-\int f d \mu\right| \leq \epsilon \mu(U), \quad\left|\limsup _{d \in D} \int f d \mu_{d}-\int f d \mu\right| \leq \epsilon \mu(U) .
$$

In the same way, one can prove

$$
\left|\liminf _{d \in D} \int f d \bar{\mu}_{d}-\int f d \bar{\mu}\right| \leq \epsilon \bar{\mu}(U), \quad\left|\limsup _{d \in D} \int f d \bar{\mu}_{d}-\int f d \bar{\mu}\right| \leq \epsilon \bar{\mu}(U) .
$$

Therefore, since $\epsilon$ is arbitrarily chosen, we get $\int f d \mu_{d} \rightarrow \int f d \mu$ and $\int f d \bar{\mu}_{d} \rightarrow$ $\int f d \bar{\mu}$.

Finally, let $f$ be not necessarily positive. Since $f^{+}, f^{-} \in \mathbf{M}_{0, b}(\mathcal{C}, \mathcal{U})$, by what we have just established, we get

$$
\int f d \mu_{d}=\int f^{+} d \mu_{d}-\int f^{-} d \bar{\mu}_{d} \rightarrow \int f^{+} d \mu-\int f^{-} d \bar{\mu}=\int f d \mu .
$$

(vi) $\Rightarrow$ (i). It is enough to follow the same reasoning as in the previous step by letting $C^{\prime} \in \mathcal{R}_{\mu}^{0} \cap \mathcal{R}_{\bar{\mu}}^{0}$ instead of $U \in \mathcal{R}_{\mu}^{0} \cap \mathcal{R}_{\bar{\mu}}^{0}$ and $C_{i}=\left\{f \geq t_{i}\right\}$ instead of $U_{i}=\left\{f>t_{i}\right\}(i=1, \ldots, m)$. 
Remark 3.5. In the characterization theorem, without any other hypothesis, the conjugate set functions can not be dropped from statements (iii), (iv), (v) and (vi). Indeed, let $\Omega$ be the set of natural numbers, $\mathcal{C}$ the family of finite subsets and $\mathcal{U}$ the power set $2^{\Omega}$. Then, $\mathbf{M}_{0, b}(\mathcal{C}, \mathcal{U})$ is the set of real maps with finite support and hence the separation property holds (given $C \subset U$, consider the indicator function $1_{C}$ ). Now, for any natural $n$, consider the monotone set function $\mu_{n}$ such that $\mu_{n}(\Omega)=n$ and $\mu_{n}(F)=0$ when $F \neq \Omega$. Therefore, $\bar{\mu}_{n}(F)=\left\|\mu_{n}\right\|-\mu_{n}\left(F^{c}\right)=n$ for any $F \neq \emptyset$. Moreover, $\mathcal{R}_{\mu_{n}}^{0}=2^{\Omega}-\{\Omega\}$ and $\mathcal{R}_{\bar{\mu}_{n}}^{0}=2^{\Omega}-\{\emptyset\}$. For any proper subset $F$, we have $\mu_{n}(F) \rightarrow \mu_{1}(F)$. On the other hand, given $F \neq \emptyset$, we have $\bar{\mu}_{n}(F) \rightarrow \infty \neq 1=\bar{\mu}_{1}(F)$. To better understand why that happens (in the light of the characterization theorem), consider the function $f=-1_{\{1\}}$ and observe that, by Theorem A.4(i),(iii), we have $\int f d \mu_{n}=-\int 1_{\{1\}} d \bar{\mu}_{n}=-\bar{\mu}_{n}(\{1\})=-n \rightarrow-\infty \neq-1=\int f d \mu_{1}$ (i.e. the sequence $\left(\mu_{n}\right)$ is not weakly convergent to $\left.\mu_{1}\right)$.

Going through the proof of the characterization theorem, one easily gets the following result which characterizes the convergence of integrals w.r.t. the positive cone of $\mathbf{M}_{0, b}(\mathcal{C}, \mathcal{U})$.

Theorem 3.6. Given $\mu$ and a net $\left\{\mu_{d} ; d \in D\right\}$, the following statements are equivalent:

(i) $\int f d \mu_{d} \rightarrow \int f d \mu$ for any $f \in \mathbf{M}_{0, b}(\mathcal{C}, \mathcal{U})$ such that $f \geq 0$;

(ii) $\int f d \bar{\mu}_{d} \rightarrow \int f d \bar{\mu}$ for any $f \in \mathbf{M}_{0, b}(\mathcal{C}, \mathcal{U})$ such that $f \leq 0$;

(iii) $\lim \sup _{d \in D} \mu_{d}(C) \leq \mu(C), \liminf _{d \in D} \mu_{d}(U) \geq \mu(U)$ for all $C, U \in \mathcal{R}_{\mu}$;

(iv) $\mu_{d}(F) \rightarrow \mu(F)$ for all $F \in \mathcal{R}_{\mu}^{0}$;

(v) $\mu_{d}(U) \rightarrow \mu(U)$ for all $U \in \mathcal{R}_{\mu}^{0} \cap \mathcal{R}_{\mu}^{0}$;

(vi) $\mu_{d}(C) \rightarrow \mu(C)$ for all $C \in \mathcal{R}_{\mu}^{0} \cap \mathcal{R}_{\mu}^{0}$.

In the light of Remark 3.5, it is interesting to study when it is possible to drop the conjugate set functions in the characterization of the weak convergence. The following theorem points out a simple and natural condition assuring that.

Theorem 3.7. Let $\mathcal{C}=\mathcal{U}^{c}$. Then, given $\mu$ and a net $\left\{\mu_{d} ; d \in D\right\}$, the following statements are equivalent:

(i) $\mu_{d} \stackrel{w}{\rightarrow} \mu$; 
(ii) $\bar{\mu}_{d} \stackrel{w}{\rightarrow} \bar{\mu}$;

(iii) $\lim \sup _{d \in D} \mu_{d}(C) \leq \mu(C), \liminf _{d \in D} \mu_{d}(U) \geq \mu(U)$ for all $C, U \in \mathcal{R}_{\mu}$;

(iv) $\mu_{d}(F) \rightarrow \mu(F)$ for all $F \in \mathcal{R}_{\mu}^{0}$;

(v) $\mu_{d}(U) \rightarrow \mu(U)$ for all $U \in \mathcal{R}_{\mu}^{0} \cap \mathcal{R}_{\mu}^{0}$;

(vi) $\mu_{d}(C) \rightarrow \mu(C)$ for all $C \in \mathcal{R}_{\mu}^{0} \cap \mathcal{R}_{\bar{\mu}}^{0}$.

Proof. Since $\Omega \in \mathcal{C}$ (recall $\emptyset \in \mathcal{U}$ ), the set $\Omega$ is strongly regular w.r.t. any bounded monotone set function.

First, we prove that (iii) implies statement (iii) of the characterization theorem. On noting that $\Omega \in \mathcal{C} \cap \mathcal{U}$, we have $\|\mu\| \leq \liminf _{d \in D}\left\|\mu_{d}\right\| \leq$ $\lim \sup _{d \in D}\left\|\mu_{d}\right\| \leq\|\mu\|$ and hence $\left\|\mu_{d}\right\| \rightarrow\|\mu\|$. Given $C \in \mathcal{R}_{\bar{\mu}}$, by Remark 2.2(i), we have $C^{c} \in \mathcal{R}_{\mu} \cap \mathcal{U}$ and hence

$$
\begin{aligned}
\limsup _{d \in D} \bar{\mu}_{d}(C) & =\limsup _{d \in D}\left(\left\|\mu_{d}\right\|-\mu_{d}\left(C^{c}\right)\right) \\
& \leq \limsup _{d \in D}\left\|\mu_{d}\right\|-\liminf _{d \in D} \mu_{d}\left(C^{c}\right) \leq\|\mu\|-\mu\left(C^{c}\right)=\bar{\mu}(C) .
\end{aligned}
$$

In a similar way, one can prove that $\liminf _{d \in D} \bar{\mu}_{d}(U) \geq \bar{\mu}(U)$ for any $U \in \mathcal{R}_{\bar{\mu}}$.

Now, we prove that (iv) implies statement (iv) of the characterization theorem. Since $\Omega \in \mathcal{R}_{\mu}^{0}$, we have $\left\|\mu_{d}\right\| \rightarrow\|\mu\|$. Given $F \in \mathcal{R}_{\mu}^{0}$, by Remark 2.2(i), we have $F^{c} \in \mathcal{R}_{\mu}^{0}$ and hence $\bar{\mu}_{d}(F)=\left\|\mu_{d}\right\|-\mu_{d}\left(F^{c}\right) \rightarrow\|\mu\|-\mu\left(F^{c}\right)=$ $\bar{\mu}(F)$.

Finally, we prove that (v) implies statement (v) of the characterization theorem. Since $\Omega \in \mathcal{U} \cap \mathcal{R}_{\mu}^{0} \cap \mathcal{R}_{\bar{\mu}}^{0}$, we have $\left\|\mu_{d}\right\| \rightarrow\|\mu\|$. Given $C \in \mathcal{R}_{\bar{\mu}}^{0} \cap \mathcal{R}_{\bar{\mu}}^{0}=$ $\mathcal{R}_{\mu}^{0} \cap \mathcal{R}_{\bar{\mu}}^{0}$ (recall $\overline{\bar{\mu}}=\mu$ ), by Remark 2.2(i), we have $C^{c} \in \mathcal{U} \cap \mathcal{R}_{\mu}^{0} \cap \mathcal{R}_{\bar{\mu}}^{0}$ and hence $\bar{\mu}_{d}(C)=\left\|\mu_{d}\right\|-\mu_{d}\left(C^{c}\right) \rightarrow\|\mu\|-\mu\left(C^{c}\right)=\bar{\mu}(C)$. Consequently, by the equivalence (v) $\Longleftrightarrow$ (vi) in Theorem 3.6 (take $\bar{\mu}_{d}, \bar{\mu}$ as $\mu_{d}, \mu$ respectively), we have $\bar{\mu}_{d}(U) \rightarrow \bar{\mu}(U)$ for any $U \in \mathcal{R}_{\mu}^{0} \cap \mathcal{R}_{\mu}^{0}$.

In a similar way, one can prove that (vi) implies statement (vi) of the characterization theorem.

To conclude the section we supply a result which generalizes Theorem 3.3 in Girotto and Holzer [7] (recall Remark 2.2(ii), Remark 3.2(i) and Theorem A.11).

Theorem 3.8. Let $\mathcal{C}=\mathcal{U}^{c}$. Moreover, given $\mu$ and a net $\left\{\mu_{d} ; d \in D\right\}$, let $\mu, \mu_{d}$ be additive set functions for any $d \in D$. Then, the following statements are equivalent: 
(i) $\mu_{d} \stackrel{w}{\rightarrow} \mu$;

(ii) $\left\|\mu_{d}\right\| \rightarrow\|\mu\|$ and $\lim \sup _{d \in D} \mu_{d}(C) \leq \mu(C)$ for all $C \in \mathcal{R}_{\mu}$;

(iii) $\left\|\mu_{d}\right\| \rightarrow\|\mu\|$ and $\liminf \operatorname{in}_{d \in D} \mu_{d}(U) \geq \mu(U)$ for all $U \in \mathcal{R}_{\mu}$;

(iv) $\mu_{d}(F) \rightarrow \mu(F)$ for all $F \in \mathcal{R}_{\mu}^{0}$;

(v) $\mu_{d}(U) \rightarrow \mu(U)$ for all $U \in \mathcal{R}_{\mu}^{0}$;

(vi) $\mu_{d}(C) \rightarrow \mu(C)$ for all $C \in \mathcal{R}_{\mu}^{0}$.

Proof. We start proving that (ii) implies statement (iii) of the previous theorem. Let $U \in \mathcal{R}_{\mu}$. Then, by the additivity assumption and Remark 2.2(i), we have $U^{c} \in \mathcal{R}_{\mu} \cap \mathcal{C}(\mu=\bar{\mu}$ !) and hence

$$
\begin{aligned}
\liminf _{d \in D} \mu_{d}(U) & =\liminf _{d \in D}\left(\left\|\mu_{d}\right\|-\mu_{d}\left(U^{c}\right)\right) \\
& \geq \liminf _{d \in D}\left\|\mu_{d}\right\|-\limsup _{d \in D} \mu_{d}\left(U^{c}\right)=\|\mu\|-\limsup _{d \in D} \mu_{d}\left(U^{c}\right) \\
& \geq\|\mu\|-\mu\left(U^{c}\right)=\mu(U),
\end{aligned}
$$

where the second equality follows from $\left\|\mu_{d}\right\| \rightarrow\|\mu\|$.

In a similar way, one can verify that (iii) implies statement (iii) of the previous theorem.

\section{The Lévy-Topology and a Prokhorov Type Theorem}

In order to use the machinery of general topology to investigate the properties of weak convergence, in the following definition, suggested by the characterization theorem, we introduce the Lévy-topology.

Definition 4.1. The Lévy-topology on the $\operatorname{set} \operatorname{bm}(\Omega, \mathcal{F})$ of bounded monotone set functions on $\mathcal{F}$ is the topology such that, for any $\mu$, the basic neighborhoods of $\mu$ are the sets of the form:

$\mathbf{N}_{\epsilon, F_{1}, \ldots, F_{k}}(\mu)=\left\{\mu^{\prime}:\left|\mu^{\prime}\left(F_{i}\right)-\mu\left(F_{i}\right)\right|<\epsilon,\left|\bar{\mu}^{\prime}\left(F_{i}\right)-\bar{\mu}\left(F_{i}\right)\right|<\epsilon \quad(i=1, \ldots, k)\right\}$, where $\epsilon>0$ and $F_{1}, \ldots, F_{k}$ are elements of $\mathcal{R}_{\mu}^{0} \cap \mathcal{R}_{\mu}^{0}$.

The next result easily follows from the characterization theorem and links weak convergence of bounded monotone set functions and convergence under the Lévy-topology. 
Theorem 4.2. Given $\mu$ and a net $\left\{\mu_{d} ; d \in D\right\}$, the following statements are equivalent:

(i) $\mu_{d} \stackrel{w}{\rightarrow} \mu$;

(ii) $\mu_{d}$ converges to $\mu$ under the Lévy-topology.

In the sequel, we call bounded any set or any net which is in the ball $\operatorname{bm}^{(\leq r)}(\Omega, \mathcal{F})=\{\mu:\|\mu\| \leq r\}$ for some real number $r$. Moreover, we call surface of $\mathrm{bm}^{(\leq r)}(\Omega, \mathcal{F})$ the set $\mathrm{bm}^{(r)}(\Omega, \mathcal{F})=\{\mu:\|\mu\|=r\}$. The following theorem assures that any bounded set of monotone set functions is relatively compact.

Theorem 4.3. Any bounded net in $\operatorname{bm}(\Omega, \mathcal{F})$ has a subnet pointwise convergent (and hence weakly convergent) to a bounded monotone set function.

Proof. Let $\left\{\mu_{d} ; d \in D\right\}$ be a net in $\operatorname{bm}^{(\leq r)}(\Omega, \mathcal{F}) \subset[0, r]^{\mathcal{F}}=X$. Since, by Tychonoff Theorem, the set $X$ is a compact space w.r.t. the product topology, there are a subnet $\left\{\mu_{d^{\prime}} ; d^{\prime} \in D^{\prime} \subset D\right\}$ and a set function $\nu \in X$ such that $\nu(F)=\lim _{d^{\prime} \in D^{\prime}} \mu_{d^{\prime}}(F)$ for any $F$. Consequently, $\nu \in \operatorname{bm}(\Omega, \mathcal{F})$ and

$$
\begin{aligned}
\bar{\nu}(F) & =\nu(\Omega)-\nu\left(F^{c}\right)=\lim _{d^{\prime} \in D^{\prime}} \mu_{d^{\prime}}(\Omega)-\lim _{d^{\prime} \in D^{\prime}} \mu_{d^{\prime}}\left(F^{c}\right) \\
& =\lim _{d^{\prime} \in D^{\prime}}\left[\mu_{d^{\prime}}(\Omega)-\mu_{d^{\prime}}\left(F^{c}\right)\right]=\lim _{d^{\prime} \in D^{\prime}} \bar{\mu}_{d^{\prime}}(F)
\end{aligned}
$$

for any $F$. Therefore, by the characterization theorem 3.4 , we get $\mu_{d^{\prime}} \stackrel{w}{\rightarrow} \nu$.

The following result assures that the Lévy-topology is locally convex and $\sigma$-compact.

Theorem 4.4. For any $r>0$, the sets $\operatorname{bm}^{(\leq r)}(\Omega, \mathcal{F})$ and $\operatorname{bm}^{(r)}(\Omega, \mathcal{F})$ are compact. Therefore, the topological space $\operatorname{bm}(\Omega, \mathcal{F})$ is locally convex and $\sigma$ compact.

Proof. Since any basic neighborhood of the Lévy-topology is a convex set, the topological space $\operatorname{bm}(\Omega, \mathcal{F})$ is locally convex.

Now, given $r>0$, let $\left\{\mu_{d} ; d \in D\right\}$ be a net in $\operatorname{bm}^{(\leq r)}(\Omega, \mathcal{F})$. Then, by Theorem 4.3, there is a subnet $\left\{\mu_{d^{\prime}} ; d^{\prime} \in D^{\prime} \subset D\right\}$ which is pointwise convergent to some $\mu$. Therefore, $\left\|\mu_{d^{\prime}}\right\|=\mu_{d^{\prime}}(\Omega) \rightarrow \mu(\Omega)=\|\mu\|$. Since $\left\|\mu_{d^{\prime}}\right\| \leq r$ for any $d^{\prime} \in D^{\prime}$, we have $\|\mu\| \leq r$. Consequently, the set $\operatorname{bm}^{(\leq r)}(\Omega, \mathcal{F})$ is compact (by similar arguments, the same holds for $\operatorname{bm}^{(r)}(\Omega, \mathcal{F})$ ). This assures the $\sigma$-compactness of $\operatorname{bm}(\Omega, \mathcal{F})$.

Theorem 4.5. Let $\Omega \in \mathcal{C}$. Then $\mathrm{bm}^{(\leq r)}(\Omega, \mathcal{F})$ and $\mathrm{bm}^{(r)}(\Omega, \mathcal{F})$ are closed for any real $r>0$. Therefore, the topological space $\operatorname{bm}(\Omega, \mathcal{F})$ is $\sigma$-compact, locally convex and locally compact. 
Proof. Since $\Omega \in \mathcal{C}$, we have $\Omega \in \mathcal{R}_{\mu}^{0} \cap \mathcal{R}_{\bar{\mu}}^{0}$ for all $\mu$. Given $r>0$, first we verify that $\operatorname{bm}^{(\leq r)}(\Omega, \mathcal{F})$ is closed. Let $\left\{\mu_{d} ; d \in D\right\}$ be a net in $\operatorname{bm}^{(\leq r)}(\Omega, \mathcal{F})$ such that $\mu_{d} \stackrel{w}{\rightarrow} \mu$. Then, by the characterization theorem, $\left\|\mu_{d}\right\|=\mu_{d}(\Omega) \rightarrow \mu(\Omega)=\|\mu\|$ and hence $\|\mu\| \leq r$, so that $\mu \in \operatorname{bm}^{(\leq r)}(\Omega, \mathcal{F})$. Therefore, bm ${ }^{(\leq r)}(\Omega, \mathcal{F})$ is closed (by similar arguments, the same holds for $\left.\mathrm{bm}^{(r)}(\Omega, \mathcal{F})\right)$. In order to verify the local compactness, given $\mu_{0}$, choose $\epsilon>0$ and consider the basic neighborhood $\mathbf{N}_{\epsilon, \Omega}\left(\mu_{0}\right)=\left\{\mu:\left|\|\mu\|-\left\|\mu_{0}\right\|\right|<\epsilon\right\}$. Then, $\mathbf{N}_{\epsilon, \Omega}\left(\mu_{0}\right) \subset$ bm $^{\left(\leq\left\|\mu_{0}\right\|+\epsilon\right)}(\Omega, \mathcal{F})$ and hence, by the previous part of the proof and Theorem 4.3, the closure of $\mathbf{N}_{\epsilon, \Omega}\left(\mu_{0}\right)$ is compact. Keeping the previous theorem in mind, completes the proof.

In order to get an extension of Prokhoroff Theorem in the context of bounded monotone set functions, we introduce the notion of tightness w.r.t. a fixed non empty subset $\mathcal{K}$ of $\mathcal{C}$ (the elements of $\mathcal{K}$ will be denoted by $K$, with or without indices).

Definition 4.6. (i) A bounded monotone set function $\mu$ is called:

- $\mathcal{K}$-tight iff for any $\epsilon>0$ there is $K$ such that $\|\mu\|-\mu(K)=\bar{\mu}\left(K^{c}\right)<\epsilon$;

- strongly $\mathcal{K}$-tight iff for any $\epsilon>0$ there is $K \in \mathcal{R}_{\mu}^{0} \cap \mathcal{R}_{\bar{\mu}}^{0}$ such that $\bar{\mu}\left(K^{c}\right)<\epsilon$.

We denote by $\operatorname{tm}(\Omega, \mathcal{F}, \mathcal{K})$ the set of $\mathcal{K}$-tight bounded monotone set functions.

(ii) A subset $\mathbf{S}$ of $\operatorname{tm}(\Omega, \mathcal{F}, \mathcal{K})$ is called uniformly $\mathcal{K}$-tight iff for any $\epsilon>0$ there is $K$ such that $\sup \left\{\bar{\mu}\left(K^{c}\right): \mu \in \mathbf{S}\right\}<\epsilon$.

Theorem 4.7. (Prokhoroff type theorem) The following statements hold:

(i) Let $\mathbf{S}$ be a subset of $\operatorname{bm}(\Omega, \mathcal{F})$ closed under pointwise convergence. Then, any bounded uniformly $\mathcal{K}$-tight subset $\mathbf{S}_{0}$ of $\mathbf{S}$ is relatively compact in the subspace $\mathbf{S} \cap \operatorname{tm}(\Omega, \mathcal{F}, \mathcal{K})$ (the direct form);

(ii) Let $\Omega \in \mathcal{C}$. Moreover let $(\mathcal{K}, \supset)$ be a directed set. Then, any compact set of strongly $\mathcal{K}$-tight bounded monotone set functions is uniformly $\mathcal{K}$-tight (the converse form).

Proof. (i) Let $\mathbf{S}_{0}$ be a bounded uniformly $\mathcal{K}$-tight subset of $\mathbf{S}$. Given a net $\left\{\mu_{d} ; d \in D\right\}$ in $\mathbf{S}_{0}$, by Theorem 4.3 , there is a subnet $\left\{\mu_{d^{\prime}} ; d^{\prime} \in D^{\prime} \subset D\right\}$ which is pointwise convergent to some $\mu$. Since $\mathbf{S}$ is closed under pointwise convergence, we have $\mu \in \mathbf{S}$. In order to verify that $\mu \in \operatorname{tm}(\Omega, \mathcal{F}, \mathcal{K})$, note that $\left\|\mu_{d^{\prime}}\right\| \rightarrow\|\mu\|$. Consequently, given $\epsilon>0$, there is $d_{1} \in D^{\prime}$ such that $\left|\left\|\mu_{d^{\prime}}\right\|-\|\mu\|\right|<\epsilon$ for any $d^{\prime} \geq_{D} d_{1}$. Moreover, since $\mathbf{S}_{0}$ is uniformly $\mathcal{K}$-tight, there is $K$ such that $\left\|\mu_{d^{\prime}}\right\|-\mu_{d^{\prime}}(K)<\epsilon$ for any $d^{\prime} \in D^{\prime}$. Finally, there 
is $d_{2} \in D^{\prime}$ such that $\left|\mu_{d^{\prime}}(K)-\mu(K)\right|<\epsilon$ for any $d^{\prime} \geq_{D} d_{2}$. Then, given $d^{\prime} \geq_{D} d_{1} \vee d_{2}$, we have

$$
\|\mu\|-\mu(K) \leq\left|\|\mu\|-\left\|\mu_{d^{\prime}}\right\|\right|+\left|\| \mu_{d^{\prime}}\right|\left|-\mu_{d^{\prime}}(K)\right|+\left|\mu_{d^{\prime}}(K)-\mu(K)\right|<3 \epsilon .
$$

Hence, $\mu \in \operatorname{tm}(\Omega, \mathcal{F}, \mathcal{K})$. This completes the proof of the direct half of the theorem.

(ii) Since $\Omega \in \mathcal{C}$, we have $\Omega \in \mathcal{R}_{\mu}^{0} \cap \mathcal{R}_{\mu}^{0}$ for all $\mu$. Let $\mathbf{S}$ be a compact set of strongly $\mathcal{K}$-tight bounded monotone set functions. Let $\epsilon>0$. Then, for any $\mu \in \mathbf{S}$, there is $K_{\mu} \in \mathcal{R}_{\mu}^{0} \cap \mathcal{R}_{\mu}^{0}$ such that $\|\mu\|-\mu\left(K_{\mu}\right)<\epsilon$. Now, for any $\mu \in \mathbf{S}$, consider the neighborhood:

$$
\begin{gathered}
\mathbf{N}_{\epsilon, \Omega, K_{\mu}}(\mu)=\left\{\mu^{\prime}:||\left|\mu^{\prime}\|-\| \mu \|\right|<\epsilon,\left|\mu^{\prime}\left(K_{\mu}\right)-\mu\left(K_{\mu}\right)\right|<\epsilon\right. \\
\left.\left|\bar{\mu}^{\prime}\left(K_{\mu}\right)-\bar{\mu}\left(K_{\mu}\right)\right|<\epsilon\right\} .
\end{gathered}
$$

The family of these neighborhoods is an open cover of $\mathbf{S}$ and hence there is a finite subcover $\mathbf{N}_{\epsilon, \Omega, K_{\mu_{i}}}\left(\mu_{i}\right)(i=1, \ldots, n)$. Since $(\mathcal{K}, \supset)$ is a directed set, there is $K \supset \bigcup_{i=1}^{n} K_{\mu_{i}}$. Now, given $\mu \in \mathbf{S}$, there is $i \in\{1, \ldots, n\}$ such that $\mu \in \mathbf{N}_{\epsilon, \Omega, K_{\mu_{i}}}\left(\mu_{i}\right)$ and hence we have

$$
\begin{aligned}
\|\mu\|-\mu(K) & \leq\|\mu\|-\mu\left(K_{\mu_{i}}\right) \\
& \leq\left|\|\mu\|-\left\|\mu_{i}\right\|\right|+\left|\left\|\mu_{i}\right\|-\mu_{i}\left(K_{\mu_{i}}\right)\right|+\left|\mu_{i}\left(K_{\mu_{i}}\right)-\mu\left(K_{\mu_{i}}\right)\right| \\
& <3 \epsilon .
\end{aligned}
$$

Remark 4.8. (i) With reference to the direct form of Prokhorov type theorem, if the induced Lévy-topology on the subspace $\mathbf{S}$ is metrizable, then we can assume $\mathbf{S}$ to be closed under pointwise convergence of sequences.

(ii) Let $\Omega$ be a separable metric space, $\mathcal{F}$ the Borel $\sigma$-field and $\mathcal{C}, \mathcal{U}, \mathcal{K}$ the families of closed, open and compact sets, respectively. Moreover, let $\mathbf{S}=\mathrm{ca}_{+}(\Omega, \mathcal{F})$ the subset of positive measures on $\mathcal{F}$. Then, by metrization theorem 3.1 in Varadarajan [10], the induced Lévy-topology on the subspace $\mathbf{S}$ is metrizable as well. Moreover, by Vitali-Hahn-Saks theorem, $\mathbf{S}$ is closed under pointwise convergence of sequences. Consequently, by (i) and Theorem 4.7(i), we get the well known direct form of Prokhorov theorem.

(iii) Let $\mathcal{F}$ be a field on $\mathbf{S}=\mathrm{ba}_{+}(\Omega, \mathcal{F})$ the subset of masses (i.e. positive bounded charges) on $\mathcal{F}$. Since $\mathbf{S}$ is closed under pointwise convergence, Theorem 4.7(i) assures that any bounded uniformly $\mathcal{K}$-tight set of masses is relatively compact in the subspace of tight masses.

(iv) Let $\Omega$ be a locally compact normal space and $\mathcal{C}, \mathcal{U}, \mathcal{K}$ the families of closed, open and compact closed sets, respectively. Then, any compact set of $\mathcal{K}$-tight bounded monotone set functions is uniformly $\mathcal{K}$-tight. To see this, by 
the converse form of Prokhorov type theorem, it is enough to prove that any $\mathcal{K}$-tight $\mu$ is strongly $\mathcal{K}$-tight. Let $\mu$ be $\mathcal{K}$-tight. Then, given $\epsilon>0$, there is $K \in \mathcal{C}$ such that $\bar{\mu}\left(K^{c}\right)<\epsilon$. Since $\Omega$ is locally compact, by Theorem 3.3.2 in Engelking [5], there is $K^{\prime} \in \mathcal{C}$ such that $K \subset \stackrel{o}{K^{\prime}}$. Then, by Lemma 3.3, there is $C \in \mathcal{R}_{\mu}^{0} \cap \mathcal{R}_{\mu}^{0}$ such that $K \subset C \subset \stackrel{o}{K^{\prime}}$. Consequently, $C^{c} \subset K^{c}$ and hence $\bar{\mu}\left(C^{c}\right) \leq \bar{\mu}\left(K^{c}\right)<\epsilon$; moreover, since $C$ is a closed subset of the compact $K^{\prime}$, we have $C \in \mathcal{K}$.

\section{A The Choquet Integral}

We supply here some properties of the Choquet integral which are instrumental in proving some results of the paper. We recall that one can find an extensive treatment of this integral in Denneberg [4] (called there asymmetric integral). Now, we start with the following definition.

Definition A.1. A real map $f$ on $\Omega$ is said to be Choquet-measurable (w.r.t. $\mathcal{F})$ iff $\left\{f^{+}>t\right\},\left\{f^{-}>t\right\} \in \mathcal{F}$ for any real $t \geq 0$. Moreover, $f$ is called a simple function if it is Choquet-measurable and its image is finite.

Note that the sum of Choquet-measurable maps may fail to be Choquetmeasurable, so that the sum of simple functions is not necessarily simple. Now, we give two elementary characterizations of simple functions.

Remark A.2. (i) A non negative real map $f$ is a simple function iff $f=$ $\sum_{i=1}^{n} c_{i} 1_{F_{i}}$ with $c_{1}, \ldots, c_{n} \geq 0$ and $F_{1} \supset \cdots \supset F_{n}$.

(ii) Let $\mathcal{F}$ be a field. Then any real map $f$ is a simple function iff $f=$ $\sum_{i=1}^{n} c_{i} 1_{F_{i}}$ with $F_{i} \cap F_{j}=\emptyset$, for any $i, j$ such that $i \neq j$. Consequently, the set of simple functions is a linear space.

Now, we come to the notion of Choquet integral. In the sequel the letter $f$, with or without indices, always denotes a Choquet-measurable map.

Definition A.3. Given $f$ and $\mu$, we denote by $\int f d \mu$ the Choquet integral of $f$ w.r.t. $\mu$, defined as:

$$
\int f d \mu= \begin{cases}\int_{0}^{\infty} \mu(\{f>t\}) d t, & \text { if } f \geq 0 \\ \int f^{+} d \mu-\int f^{-} d \bar{\mu}, & \text { otherwise }\end{cases}
$$

whenever the improper Riemann integrals $\int f^{+} d \mu$ and $\int f^{-} d \bar{\mu}$ are not both $\infty$. Moreover, the map $f$ is said to be (Choquet) $\mu$-integrable iff $\int f d \mu$ is finite (i.e. $\int f^{+} d \mu$ and $\int f^{-} d \bar{\mu}$ are both finite). 
Note that the Choquet integral always exists whenever $f$ is bounded below or bounded above; moreover, $f$ is $\mu$-integrable whenever $f$ is bounded. Now, we supply some elementary general properties of the Choquet integral (see Proposition 5.1 in Denneberg [4]).

Theorem A.4. The following statements hold:

(i) $\int 1_{F} d \mu=\mu(F)$;

(ii) $\int k f d \mu=k \int f d \mu$, for any real $k \geq 0$ (positive homogeneity);

(iii) $\int(-f) d \mu=-\int f d \bar{\mu}$ (asymmetry);

(iv) $\int(f+k) d \mu=\int f d \mu+k\|\mu\|$, for any real $k$;

(v) $\int f_{1} d \mu \leq \int f_{2} d \mu$, whenever $f_{1} \leq f_{2}$ (monotonicity);

(vi) $\int f d \mu=\int f d \nu$, for any monotone extension $\nu$ of $\mu$,

whenever any one of the terms above considered makes sense.

The previous theorem points out that the Choquet integral is positively homogeneous. Unlike S-integral and Lebesgue integral, moreover, the Choquet integral is not additive, as the following example shows.

Example A.5. Given a proper subset $A$ of $\Omega$, let $f_{1}=1_{A}, f_{2}=1_{A^{c}}$ and $\mathcal{F}$ be the smallest field on $\Omega$ including $A$. Moreover, consider $\mu$ such that $\mu(A)=$ $\mu\left(A^{c}\right)=1 / 3$ and $\|\mu\|=1$. Then $\int\left(f_{1}+f_{2}\right) d \mu=1 \neq 2 / 3=\int f_{1} d \mu+\int f_{2} d \mu$.

In order to give a sufficient condition for the additivity (see Proposition 5.1(vi) in Denneberg [4]), we recall the notion of comonotonicity which introduces an equivalence relation on the set of real maps on $\Omega$.

Definition A.6. Two real maps $g_{1}$ and $g_{2}$ on $\Omega$ are said to be comonotonic iff

$$
\left[g_{1}(\omega)-g_{1}\left(\omega^{\prime}\right)\right]\left[g_{2}(\omega)-g_{2}\left(\omega^{\prime}\right)\right] \geq 0
$$

for any $\omega, \omega^{\prime} \in \Omega$.

Remark A.7. (i) Let $g_{1}, \ldots, g_{n}$ be pairwise comonotonic and let $c_{1}, \ldots, c_{n}$, $c_{1}^{\prime}, \ldots, c_{n}^{\prime} \geq 0$. Then $\sum_{i=1}^{n} c_{i} g_{i}$ and $\sum_{i=1}^{n} c_{i}^{\prime} g_{i}$ are comonotonic as well; i.e., any equivalence class of comonotonic maps is a cone in the space of real maps on $\Omega$.

(ii) Let $F_{1} \subset F_{2}$. Then $1_{F_{1}}$ and $1_{F_{2}}$ are comonotonic. 
Theorem A.8 (Comonotonic Additivity). Let $f_{1}, \ldots, f_{n}$ be pairwise comonotonic. Then

$$
\int\left(f_{1}+\cdots+f_{n}\right) d \mu=\int f_{1} d \mu+\cdots+\int f_{n} d \mu
$$

whenever any one of the terms above considered makes sense.

Consequently, the Choquet integral is additive in each equivalence class of integrable comonotonic maps and, in any case, positively homogeneous.

Remark A.9. Let $f \geq 0$ be a simple function, i.e. $f=\sum_{i=1}^{n} c_{i} 1_{F_{i}}$ with $c_{1}, \ldots, c_{n} \geq 0$ and $F_{1} \supset \cdots \supset F_{n}$ (see Remark A.2(i)). Then, by Remark A.7, Theorem A.4(i),(ii) and Theorem A.8, we have $\int f d \mu=\sum_{i=1}^{n} c_{i} \mu\left(F_{i}\right)$ for any $\mu$.

The following result (easy consequence of the Additivity Theorem, stated in Denneberg [4]) gives a sufficient condition assuring the additivity of the Choquet integral when $\mu$ is additive.

Lemma A.10. Let $\mathcal{F}=2^{\Omega}$ and $\mu$ additive. Then

$$
\int\left(f_{1}+f_{2}\right) d \mu=\int f_{1} d \mu+\int f_{2} d \mu
$$

for any $f_{1}, f_{2}$ bounded.

We conclude this appendix by showing that, in the setting of additive set functions on a field, Choquet integral and S-integral of a bounded map $f$ are equal. Therefore, the Choquet integral w.r.t. a mass on a field is a real linear functional on the set of bounded Choquet-measurable maps.

Theorem A.11. Let $\mathcal{F}$ be a field and $\mu$ additive. Then any bounded map $f$ is S-integrable and

$$
\int f d \mu=S \int f d \mu
$$

Proof. Let $f$ be bounded. Assume first $f \geq 0$. The proof is carried out in the following two steps.

$1^{0}$. Let $f$ be a simple function. Since $\mathcal{F}$ is a field, by Remark A.2(ii), we have $f=c_{1} 1_{F_{1}}+\cdots+c_{n} 1_{F_{n}}$, where $0=c_{0}<c_{1}<\cdots<c_{n}$ and $F_{i} \cap F_{j}=\emptyset$ for any $i, j$ such that $i \neq j$. On noting that $f=\sum_{i=1}^{n}\left(c_{i}-c_{i-1}\right) 1_{\cup_{j=i}^{n} F_{j}}$, by Remark 
A.9, we get

$$
\begin{aligned}
\int f d \mu & =\sum_{i=1}^{n}\left(c_{i}-c_{i-1}\right) \mu\left(\bigcup_{j=i}^{n} F_{j}\right)=\sum_{i=1}^{n} c_{i}\left[\mu\left(\bigcup_{j=i}^{n} F_{j}\right)-\mu\left(\bigcup_{j=i+1}^{n} F_{j}\right)\right] \\
& =\sum_{i=1}^{n} c_{i} \mu\left(F_{i}\right)=\mathrm{S} \int f d \mu .
\end{aligned}
$$

$2^{0}$. Let $f$ be not necessarily simple. We claim that $f$ is the uniform limit of an increasing sequence of simple functions. Let $k=\sup f$. Given $n \geq 1$, consider the simple function $f_{n}$ defined as:

$$
f_{n}(\omega)= \begin{cases}\frac{k i}{2^{n}} & \text { if } \omega \in\left\{f>\frac{k i}{2^{n}}\right\} \cap\left\{f>\frac{k(i+1)}{2^{n}}\right\}^{c} \quad\left(i=1, \ldots, 2^{n}-1\right) \\ 0 & \text { if } \omega \in\left\{f>\frac{k}{2^{n}}\right\}^{c}\end{cases}
$$

for all $\omega \in \Omega$. Plainly, the increasing sequence $\left(f_{n}\right)$ converges to $f$ uniformly. This proves the claim.

Therefore, $f$ is $T_{1}$-measurable and hence, by Theorem 4.5.8 in Bhaskara Rao and Bhaskara Rao [2], the map $f$ is S-integrable.

Now, let $\epsilon>0$. Then, there is $n^{\prime}$ such that $0 \leq f(\omega)-f_{n}(\omega)<\epsilon$ for any $n \geq n^{\prime}$ and $\omega \in \Omega$. Hence, we get

$$
\left|\mathrm{S} \int f d \mu-\mathrm{S} \int f_{n} d \mu\right| \leq \mathrm{S} \int\left|f-f_{n}\right| d \mu \leq \mathrm{S} \int \epsilon d \mu=\epsilon\|\mu\|,
$$

for any $n \geq n^{\prime}$. Since $\epsilon$ is arbitrarily chosen, we have

$$
\mathrm{S} \int f_{n} d \mu \rightarrow \mathrm{S} \int f d \mu \text {. }
$$

In order to verify that $\int f_{n} d \mu \rightarrow \int f d \mu$, we consider a mass $\nu$ on $2^{\Omega}$, extension of $\mu$ (see Corollary 3.3.4 in Bhaskara Rao and Bhaskara Rao [2]). Now, let $\epsilon>0$. Then, there is $n^{\prime}$ such that $0 \leq f(\omega)-f_{n}(\omega)<\epsilon$ for any $n \geq n^{\prime}$ and $\omega \in \Omega$. Therefore, keeping in mind Theorem A.4, Lemma A.7 and $\bar{\nu}=\nu$ ( $\nu$ is additive!), we have

$$
\begin{aligned}
\left|\int f d \mu-\int f_{n} d \mu\right| & =\int f d \mu-\int f_{n} d \mu=\int f d \nu-\int f_{n} d \nu \\
& =\int f d \nu+\int\left(-f_{n}\right) d \bar{\nu}=\int f d \nu+\int\left(-f_{n}\right) d \nu \\
& =\int\left(f-f_{n}\right) d \nu \leq \int \epsilon d \nu=\epsilon\|\nu\|,
\end{aligned}
$$


for any $n \geq n^{\prime}$. Since $\epsilon$ is arbitrarily chosen, we have $\int f_{n} d \mu \rightarrow \int f d \mu$ so that, by $1^{0}$ and (2), we get $\int f d \mu=\mathrm{S} \int f d \mu$.

Finally, let $f$ be not necessarily positive. Then, by $2^{0}$, we have

$$
\begin{aligned}
\int f d \mu & =\int f^{+} d \mu-\int f^{-} d \bar{\mu}=\int f^{+} d \mu-\int f^{-} d \mu \\
& =\mathrm{S} \int f^{+} d \mu-\mathrm{S} \int f^{-} d \mu=\mathrm{S} \int\left(f^{+}-f^{-}\right) d \mu=\mathrm{S} \int f d \mu .
\end{aligned}
$$

\section{References}

[1] Alexandroff, D.(1940-43). Additive set functions in abstract spaces, Mat. Sb. 8, 307-348; 9, 563-628; 13, 169-238.

[2] Bhaskara Rao, K.P.S. and Bhaskara Rao, M.(1983). Theory of Charges, Academic Press, New York.

[3] Billingsley, P.(1968). Convergence of probability measures, John Wiley, New York.

[4] Denneberg, D.(1994). Non-additive measure and integral, Kluwer Academic Publisher, Dordrecht.

[5] Engelking, R.(1977). General topology, PWN-Polish Scientific Publishers, Warszawa.

[6] Girotto, B. and Holzer, S.(1993). Weak convergence of masses on normal topological spaces, Theory Probab. Appl. 37, 337-340 (an English translation of the June 1992 issue of the Soviet journal Teoriya Veroyatnostei i ee Primeneiya, 364-366).

[7] Girotto, B. and Holzer, S.(1993). Weak convergence of masses on normal topological spaces, Sankhya A 55, 188-201.

[8] Girotto, B. and Holzer, S.(2000). Weak convergence of masses: topological properties, Atti Sem. Mat. Fis. Univ. Modena 48(2), to appear.

[9] Masani, P.(1982). The outer regularization of finitely-additive measures over normal topological spaces, Proc. Measure Theory Conf., Oberwolfach, 1981, Lecture Notes in Math. 945, 116-144.

[10] Varadarajan, V.S.(1958). Weak convergence of measures on separable metric spaces, Sankhya 19, 15-22. 
